\title{
Clinical Presentations and Factors Associated with in-Hospital Mortality of Patients Diagnosed with Subacute and Chronic Infectious Meningitis in A Mexican Hospital
}

\section{Mendoza-Esquivel JF${ }^{1,2}$, Hernández-Rodríguez $\mathrm{HG}^{1,2}$, Vázquez-Herrejón $\mathrm{G}^{1,2}$, Rodríguez-Rodríguez $\mathrm{J}^{1,2}$ and Rodríguez-Leyva $\mathbf{I}^{1,2 *}$}

${ }^{1}$ Facultad de Medicina de la Universidad Autonoma de San Luis Potosí, México

${ }^{2}$ Neurology Service, Hospital Central "Dr. Ignacio Morones Prieto", San Luis Potosí, SLP, Mexico

*Corresponding Author: Rodríguez-Leyva I, 1Facultad de Medicina de la Universidad Autonoma de San Luis Potosí, México.

Received: August 26, 2019; Published: September 05, 2019

DOI: 10.31080/ASMI.2019.02.0364

\begin{abstract}
Background: Subacute/chronic meningitis are well-characterized clinical entities whose differential diagnosis is broad and challenging to address, resulting in a third of cases without an etiological diagnosis. In the world literature and especially in our country few studies describe the clinical patterns of presentation of the most common forms of subacute/chronic infectious meningitis, as well as what is the relationship of these patterns to in-hospital mortality. Cases of chronic meningitis in our environment may be over diagnosed as tuberculous meningitis because of its high regional frequency. Thus, we seek to establish the frequency of diagnostic confirmation in our region as well as identify risk factors link to in-hospital mortality.

Methods: We studied clinical records of patients with a diagnosis of chronic and subacute meningitis at discharge in five years. Clinical and auxiliary test data were analyzed to find their association with in-hospital mortality.

Results: We found that the most frequent sign in this population was acute cognitive impairment (75.5\%), followed by a headache. The majority of the cases was associated with meningeal tuberculosis. We found an association between immunosuppression, diminished level of arousal, prolonged time of disease progression, CSF acute inflammatory pattern, and hydrocephalus in the hospital mortality.
\end{abstract}

Keywords: Subacute and Chronic Meningitis; Tuberculous Meningitis; Cerebrospinal Fluid; Hydrocephalus

\section{Introduction}

Subacute and chronic infectious meningitis are well defined clinical entities although scarcely reported in medical literature [1]. While chronic meningitis is defined as a leptomeningeal inflammation with persistent neurological symptoms with cerebrospinal fluid (CSF) changes which include protein increase, lymphocytic pleocytosis and low glucose lasting 4 weeks or more, [2,3] subacute meningitis time span is not as well defined but includes those cases from five days to less than four weeks in duration not to mention shorter-lasting presentations with the aforementioned CSF changes $[4,5]$.

Independent on their temporal evolution, both entities distinguish by having typical clinical manifestations, which include headache, nuchal rigidity, photophobia, and variable grades of cognitive impairment. Fever when present is usually lower than $39^{\circ}$ Celsius. Unlike acute meningitis, signs, and symptoms in subacute/ chronic meningitis follow a more indolent course. In any case during that course, severe consciousness state deterioration, seizures, encephalitis, granulomas and abscesses, hydrocephalus, and other forms of brain, spinal cord, peripheral or cranial nerves can occur $[4,6]$.

Subacute and chronic meningitis are less frequent than their acute counterpart, and that is why we do not know their precise incidence rates [7]. In addition to this, there are other epidemiological aspects which hinder their study including the diverse etiological causes by geographical region $[8,9]$

Diagnosis for this type of meningitis is extensive and comprise many forms of infectious and non-infectious diseases (Table 1). The most frequent infectious chronic meningitis is caused by Mycobacterium tuberculosis, fungal agents mainly cryptococcus, some virus, syphilis, and Lyme disease. It can also be seen in some parasitic infections such as neurocysticercosis. 


\begin{tabular}{|l|l|}
\hline \multicolumn{1}{|c|}{ Infectious causes } & \multicolumn{1}{c|}{ Non infectious } \\
\hline Bacterial: Tuberculosis & Neoplastic: Carcinomatosis \\
Listeriosis & Lymphomatosis \\
Brucellosis & Sarcoidosis \\
Borreliosis & Sjögren Syndrome \\
Parameningeal infection & Behçet's Disease \\
\hline Fungal: Cryptococcocal & Systemic Lupus Erythematosus \\
Candidiasis & Granulomatous Angiitis \\
Coccidioidomycosis & Wegener's Granulomatosis \\
Hystoplasmosis & Vogt-Koyanagi-Harada \\
\hline $\begin{array}{l}\text { Parasitic: Cysiticercosis } \\
\text { Achantoameba }\end{array}$ & Syndrome \\
Angiostrongylus cantonensis & Migrane with CSF pleocytosis \\
Toxoplasmosis & Drug induced meningitis \\
\hline Viral: Cytomegalovirus & \\
Varicela zoster & \\
Epstein Barr Virus & \\
Herpes simplex Virus & \\
HIV & \\
Enterovirus & \\
Mumps & \\
\hline
\end{tabular}

Table 1: Causes of chronic meningitis.

Prevalence is higher in individuals with impaired cellular immunity either from disease or immunosuppressive treatment $[4,7]$. Patients in this condition may have differences in clinical presentation and evolution compared to immunocompetent hosts.

Diagnosis can be precluded by the protracted clinical course, mild symptomatology, symptomatic treatment, host immunologic state, systemic or other organ involvement. In addition isolating the causative infectious agent can be challenging by conventional means because CSF microbial colonies tend to be low and many of the causative infectious agents have slow growth rates that delay their detection by culture [4]. Therefore, diagnosis requires a meticulous clinical history including exposure, travel and immunologic state history as well as a systemic-oriented physical exam because many of the infectious agents responsible for subacute/ chronic meningitis often produce damage to other organs especially the lungs, eyes, skin, and joints. Laboratory and imaging test are always necessary, and pulmonary imaging can be useful in cases with suspicion of tuberculosis or certain mycoses with respiratory pathways of transmission.

Considering that CSF tests and neuroimaging findings are often nonspecific, diagnostic workup most often requires the more sophisticated biomolecular test. Despite this it has been estimated that a third of all chronic meningitis cases will remain without an etiologic diagnosis thus the need of a rational approach [2,5,10-13].

Delay in diagnosis can lead to subsequent delay of pathogen targeted treatment which is related to a higher morbidity and mortality risk [2,3].

Meningeal tuberculosis is the leading cause of chronic meningitis in developing countries and the more severe form of extrapulmonary tuberculosis accounting for $1-5 \%$ of these cases with mortality ranging from 7 to $45 \%[14,15]$.

In Mexico, there is an incidence of 16 cases per 100 000/year for any tuberculosis, of which $1 \%$ is meningeal with an average of 181 cases per year. Even though morbidity has been calculated as 0.1 to 0.4 per $100000 /$ year, and precise mortality rates are still unknown, estimated in-hospital mortality is $16 \%$ [16].

The adult population at risk include people from high tuberculosis prevalence, advanced age, alcoholism, patients taking immunosuppressive drugs, and oncological or HIV infected patients [17-21].

The pathologic changes seen in meningeal tuberculosis are due to basal leptomeningeal inflammation, which can produce damage to the brain parenchyma, cranial nerves, spinal cord, and nerve roots hence de different clinical presentation. Ischemic complications can arise from endarteritis and abscesses, or tuberculomas can be found. Hydrocephalus is another complication that can be resolved by repetitive lumbar puncture or shunting, by repetitive lumbar puncture or shunting, the image with CT and MRI can be essential to detect these pathological conditions and the complications [22-32].

Definitive diagnosis is reached through direct observation of acid-fast bacilli of M. tuberculosis in the CSF or tissue samples, M. Tuberculosis culture or molecular detection methods [33].

Despite adequate treatment, mortality from meningeal tuberculosis ranges between 20 and 50\% in different case series. Prognosis strongly depends on the severity of presentation [34-37].

Fungal meningitis is typical infections of immunocompromised patients, especially those with HIV infection even though they also can be found in transplant patients or those receiving immunosuppressive drugs [39]. The most common infectious agents are C. neoformans, Candida spp, and the less frequent $C$. immitis, $H$. capsulatum y B. dermatitidis. The majority of this meningitis are secondary to hematogenous dissemination of a primary lung infection $[4,40]$. 
Postmortem studies suggest that CNS mycoses are underestimated and under-diagnosed, which carry an increased mortality rate $[41,42]$. Candida meningitis mortality varies from $10 \%$ to $49 \%$. Similarly, Cryptococcus shows a mortality of up to $40 \%$ [43].

There are scarce studies, especially in our country, describing the clinical patterns of presentation of subacute/chronic meningitis, as well as the relationship between said patterns hospital mortality. Additionally, tuberculous meningitis in our background might be over diagnosed as a consequence of proper diagnostic confirmation and high regional frequency.

Based on the considerable variability of presentation between geographical regions and causative agents, it would be beneficial to recognize local clinical patterns to achieve better diagnostic and therapeutic approaches while predicting the mortality risk, helping the physician to make more efficient clinical decisions.

\section{Objective}

To describe the clinical patterns of presentation in cases diagnosed as subacute and chronic meningitis of infectious origin in our hospital, as well as determine hospital mortality to later establish the relevance of clinical patterns as mortality predictors.

\section{Materials and Methods}

We conducted a retrospective, observational medical record research in the Central Hospital in San Luis Potosí, México, of every adult patient admitted between January 2005 to December 2010 who were diagnosed with any subacute or chronic meningitis.

We included patients older than 15 years old with a complete medical record. Patients with an incomplete medical record, lack of CSF analysis, or previous chronic meningitis diagnosis were excluded.

Amongst the variables to study, we included age, gender, the presence of Diabetes, history of alcoholism, history of neoplasia, HIV infection, immunosuppressive treatment and the time lapse between symptoms start and hospital admission. We documented the presence of one or more signs/symptoms presenting at the beginning of the clinical picture and or during the first 48 hours from admission. These included fever, headache, acute cognitive impairment, changes in awareness, nausea/vomiting, meningism's, cranial nerve dysfunction, papilledema, focal signs, seizures, fever, and death.

We included CSF analysis data, presence or absence of hydrocephalus in imaging tests and if a definitive etiological diagnosis could or could not be achieved. We also considered the time be- tween hospital admission and definite diagnosis, time to beginning of treatment, and antimicrobial used as an initial treatment.

\section{Data analysis}

For the statistical analysis, we prosed the use of a statistical test based on Chi-square distribution; nevertheless, the low rate of deaths in this population, we decided to use Fisher's exact test alternatively.

\section{Results}

We searched hospital medical records of patients diagnosed with chronic or subacute meningitis, finding 49 eligible files fulfilling our inclusion criteria.

In order of frequency, diagnoses at discharge were tuberculous meningitis ( $n=35)$, chronic meningitis $(n=7)$, cryptococcal meningitis $(n=5)$, Histoplasma meningitis $(n=1)$, and coccidioides meningitis $(n=1)$, we did not find any cases of meningeal syphilis or meningeal candidiasis. Mean age for the studied population was 43.1 years (range 17 to 81 YO), mostly males (67.3\%). The total number of registered hospital deaths was 8 , representing $16.3 \%$ of all cases.

The most common background risk factor was diabetes mellitus $(28.5 \%)$, followed by alcoholism $(20.4 \%)$, all of them in male patients. $18.3 \%$ of patient's HIV positive, $6.1 \%$ was receiving immunosuppressive drugs, while $4 \%$ had a diagnosis of neoplasm.

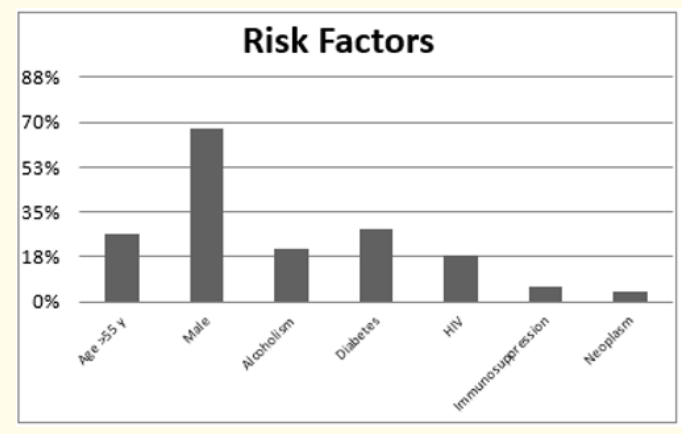

Figure 1

Symptoms at presentation were cognitive impairment (75.5\%), headache $(73.4 \%)$, vomiting $(71 \%)$, fever $(51 \%)$ average $38.5^{\circ} \mathrm{C}$, meningismus (48.9\%) altered wakefulness (44.8\%), focal signs (26\%), seizures (26\%), and last cranial nerve involvement found in 12 patients $(24.4 \%)$. The most frequently cranial nerve involved was VI CN (58\%), followed by VII, VIII, and II. Papilledema was reported in 2 cases. We must outline that $30 \%$ of studied cases presented with a combination of headache, cognitive impairment, and meningismus, all of them in the context of fever. 


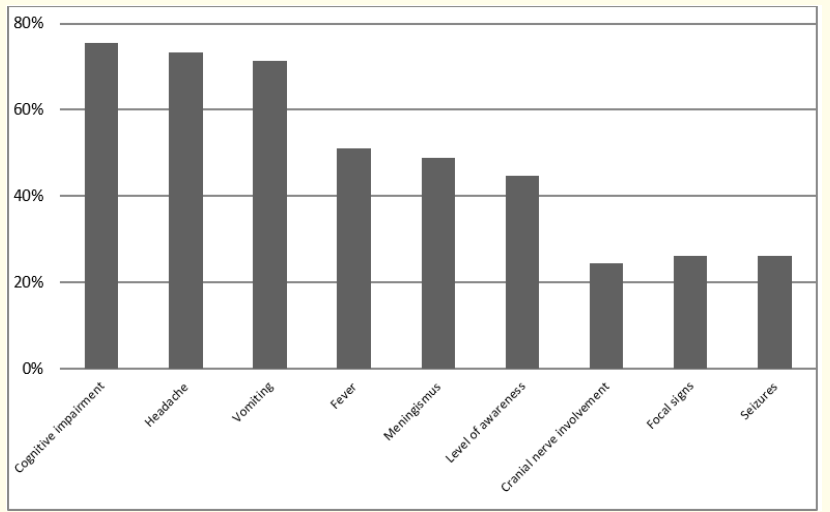

Figure 2: Clinical manifestations.

Average time from the beginning of symptoms to hospital admission was 45.4 days ( 1 to 270 days).

We encountered lymphopenia in $61 \%$ and leucocytosis in $28.5 \%$ of all complete blood count analysis. CSF findings showed low glucose in $87.8 \%$ of te samples, hyperproteinorraquia in $85.7 \%$, and high leucocyte count in $65.3 \%$ (78.1\% with lymphocyte predominance). Only $18 \%$ presented with acute inflammatory CSF changes, all of the latter with a clinical course longer than five days. We found abnormal images in $41 \%$ of patients with chest radiography $(n=43)$. Hydrocephalus was found in $34 \%$ of brain imaging.

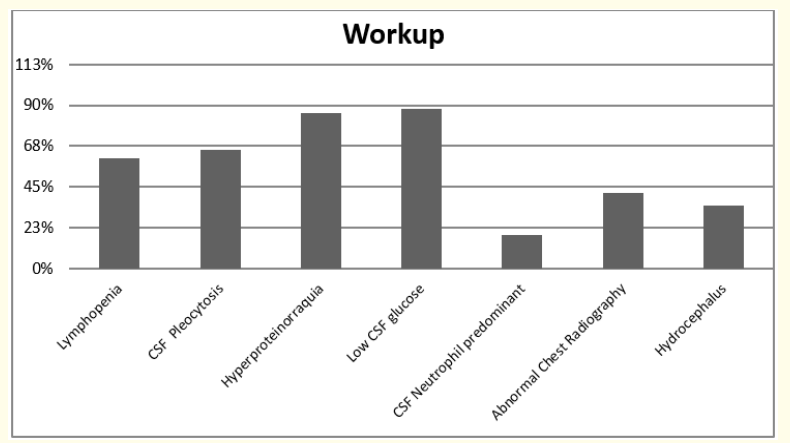

Figure 3

Etiologic diagnostic confirmation by any method was achieved in $30.6 \%(n=15)$, M. tuberculosis being the most frequent $(60 \%)$, followed by Cryptococcus (26\%), and Coccidioides (13\%). Average time to final diagnosis was 22.8 days ( 0 to 75 days), $46 \%$ of them in a period longer than 14 days. Time to diagnosis of cryptococcal meningitis was 0.8 days ( 0 to 2 days), for tuberculous men- ingitis 20.6 días ( 4 to 75 days), and of 32 and 75 days for both of the patients with meningeal coccidioidomycosis.

$81.6 \%$ of patients received empirical treatment in an average time of 7.4 days.

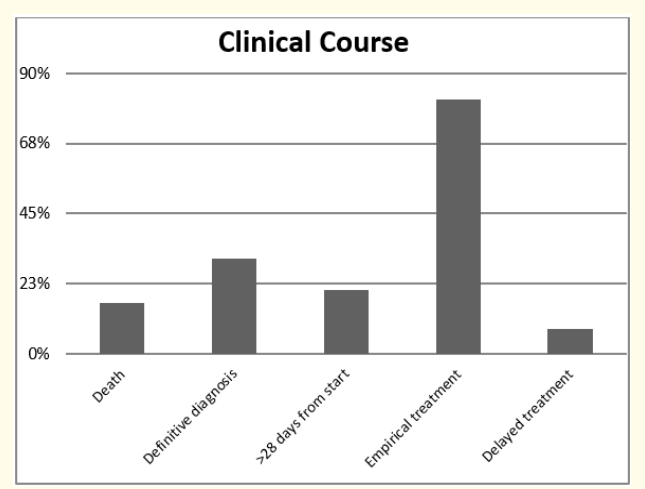

Figure 4

\section{Factors associated with mortality}

The results of clinical factors associated with death are listed in table 2.

We observed that from the eight deceased cases 5 were associated to intracranial hypertension, 1 of them also showed brainstem ischemic changes in imaging studies; one death was related to tumoral lysis syndrome, another one to septic shock, and respiratory failure in the remaining case. Even though $75 \%$ of the deaths occurred in males, a significant association between mortality and gender was not found $(\mathrm{p}=0.5)$, nor for mortality and age older than 55 years $(\mathrm{p}=0.6)$.

Separately analyzing the relationship of clinical risk factors to mortality, we found a significant association between the latter and patients with neoplasm $(\mathrm{p}=0.02)$, and patients receiving immunosuppressants $(p=0.06)$. Despite the high prevalence of diabetes mellitus in our population, we did not find any relationship between DM and mortality ( $p=0.5)$, same case for patients with HIV infection ( $p=0.5)$, or alcoholism ( $p=0.5)$.

From all the studied clinical manifestations the highest association was noted for patients with diminished level of arousal ( $p=$ $0.06)$, but no for fever ( $p=0.28)$, seizures $(p=0.88)$, cognitive impairment ( $p=0.7$ ), cranial nerve involvement ( $p=0.9$ ), vomiting (0.87), nor for a combination of headache, fever, cognitive changes and meningismus ( $\mathrm{p}=0.6)$. Regarding the time from the beginning of symptoms and its association with mortality, we used two dif- 


\begin{tabular}{|l|c|c|c|c|}
\hline \multicolumn{1}{|c|}{ Variable } & $\begin{array}{c}\text { Death } \\
\text { (n=8) }\end{array}$ & $\begin{array}{c}\text { Survivors } \\
\text { (n=41) }\end{array}$ & $\begin{array}{c}\text { \% } \\
\text { Deaths }\end{array}$ & $\boldsymbol{p}$ \\
\hline Age >55 years & $25.0 \%$ & $26.8 \%$ & $15.4 \%$ & 0.6 \\
\hline Gender & $75.0 \%$ & $65.9 \%$ & $18.2 \%$ & 0.5 \\
\hline Alcoholism & $12.5 \%$ & $22.0 \%$ & $10.0 \%$ & 0.54 \\
\hline Diabetes & $25.0 \%$ & $29.3 \%$ & $14.3 \%$ & 0.58 \\
\hline HIV & $12.5 \%$ & $19.5 \%$ & $11.1 \%$ & 0.5 \\
\hline Immunosuppression & $25.0 \%$ & $2.4 \%$ & $\mathbf{6 6 . 7 \%}$ & $\mathbf{0 . 0 6}$ \\
\hline Neoplasm & $25.0 \%$ & $0.0 \%$ & $\mathbf{1 0 0 . 0 \%}$ & $\mathbf{0 . 0 2}$ \\
\hline Cognitive impairment & $70.0 \%$ & $75.6 \%$ & $16.2 \%$ & 0.7 \\
\hline Vomiting & $50.0 \%$ & $46.3 \%$ & $17.4 \%$ & 0.87 \\
\hline Fever & $62.5 \%$ & $43.9 \%$ & $21.7 \%$ & 0.28 \\
\hline Meningismus & $66.5 \%$ & $42.3 \%$ & $20.8 \%$ & $\mathrm{~L}$ \\
\hline Diminished Arousal & $75.0 \%$ & $39.0 \%$ & $\mathbf{2 7 . 3 \%}$ & $\mathbf{0 . 0 6}$ \\
\hline $\begin{array}{l}\text { Cranial nerve } \\
\text { involvement }\end{array}$ & $37.5 \%$ & $22.0 \%$ & $25.0 \%$ & 0.91 \\
\hline Seizures & $37.5 \%$ & $24.4 \%$ & $23.1 \%$ & 0.4 \\
\hline Lymphopenia & $75.0 \%$ & $58.5 \%$ & $20.0 \%$ & 0.32 \\
\hline CSF Pleocytosis & $62.5 \%$ & $65.9 \%$ & $15.6 \%$ & 0.72 \\
\hline $\begin{array}{l}\text { Hyperproteinorra- } \\
\text { quia }\end{array}$ & $87.5 \%$ & $85.4 \%$ & $16.7 \%$ & 0.68 \\
\hline Low CSF glucose & $100.0 \%$ & $85.4 \%$ & $18.6 \%$ & 0.32 \\
\hline CSF Neutrophilia & $50.0 \%$ & $12.2 \%$ & $\mathbf{4 4 . 4 \%}$ & $\mathbf{0 . 0 2}$ \\
\hline Hydrocephalus & $75.0 \%$ & $26.8 \%$ & $\mathbf{3 5 . 3 \%}$ & $\mathbf{0 . 0 1}$ \\
\hline $\begin{array}{l}\text { Without definitive } \\
\text { diagnosis }\end{array}$ & $62.5 \%$ & $70.7 \%$ & $14.7 \%$ & 0.81 \\
\hline $\begin{array}{l}\text { Clinical course }>28 \\
\text { days }\end{array}$ & $50.0 \%$ & $14.6 \%$ & $40.0 \%$ & $\mathbf{0 . 0 4}$ \\
\hline $\begin{array}{l}\text { Without empirical } \\
\text { treatment }\end{array}$ & $12.5 \%$ & $19.5 \%$ & $11.0 \%$ & 0.54 \\
\hline Treatment delay & $0.0 \%$ & $9.8 \%$ & $0.0 \%$ & 0.47 \\
\hline
\end{tabular}

Table 2

ferent models, the first one including all cases with an evolution longer than 5 days (without association, $\mathrm{p}=0.5$ ) and a second one including only the cases with clinical course longer than 28 days this one showing statistical association $(p=0.04)$.

We did not find significant evidence between individual laboratory result another tan that documented for predominant neutrophil CSF ( $p=0.028)$ and the presence of hydrocephalus $(p=0.01)$.

Lacking a definitive etiological diagnosis did not increase mortality $(p=0.6)$, neither the delaying treatment $(p=0.47)$ or not receiving any empirical treatment $(p=0.54)$.

\section{Discussion}

Chronic and subacute meningitis are clinical entities associated with high mortality rates, facilitated by difficulties for reaching a precise etiological diagnosis early in the course of the disease. In Mexico, we often lack resources to reach that goal. That is why we must try to comprehend the most frequent clinical patterns of presentation in patients with chronic meningitis in an attempt to identify changes that could orient diagnosis to a particular pathogen.

Medical literature reports that that one third part of patients with this condition will remain without a definitive diagnosis, but in our study, we found that in our population two thirds of the patients were discharged without an etiological diagnosis [35-40].

The inability to reach a proper diagnosis generates delays in treatment start, which in turn can increase mortality, in the same way, that it has been observed in immunocompromised and more prolonged disease duration. In this study, we tried to establish the frequency for hospital mortality under the hypothesis that it would be higher in immunocompromised patients and those with a more prolonged disease, those lacking a precise diagnosis or delays in treatment [45-47]. We also analyzed the different forms of clinical presentations in this heterogeneous group of meningitis to recognize patterns associated to mortality looking to, in the future, shorten the time to treatment start and achieve better outcomes for these patients [48-53,54]. This point is critical because in places where technological and advanced diagnostic tools are unavailable clinical features analysis becomes essential.

In the epidemiological analysis of factors associated with immunocompromise we found a prevalence of DM higher than the general population (28.5\% Vs $7.5-12 \%$ ), from which we can infer that in truth the population carrying DM is more likely to acquire this type of infections, However, it was not possible to establish an association with mortality, same with patients with HIV. On the contrary, patients with immunotherapy and neoplasm showed a significant association with in-hospital mortality; however, this relationship could be masked by the severity of the underlying disease, as well as by the fact that 1 of 3 patients with immunotherapy received this as part of treatment for neoplasia.

We assumed that patients with a longer evolution time would have a higher complication rate and therefore a higher in-hospital associated mortality, however, this relationship could not be demonstrated when cases with 5 days or more of evolution were included, however, when mortality was analyzed in cases with more than 28 days of evolution, the existence of the association was demonstrated. 
Possibly because the slowly progressive and indolent natural course of the disease, higher mortality rates could be observed at a longer follow-up time. It would be necessary to design a long-term prospective study in order to verify this statement.

Regarding treatment, we observed that the majority of patients received empirical treatment, initiated in a short time. No relationship was established between delay in treatment initiation or absence of empirical treatment with mortality. There is no way to know if the absence of a relationship derives from adequate and early empirical treatment since no precise diagnosis was established in the majority of patients. It may also be because the evolution of these patients at discharge was not observed. What was observed is that the most used empirical treatment was against tuberculosis, probably because tuberculosis is often the first diagnostic option. The clinical findings associated with in-hospital mortality in this population were mostly those associated with intracranial hypertension, which was ultimately the cause of death in most cases. We mentioned that the patients most likely to die during hospitalization were those with evidence of hydrocephalus as well as patients who presented with altered wakefulness. The association found between CSF with a predominance of polymorphonuclear leukocytes and mortality could be explained by the aggressive inflammatory response of the host, causing significant damage due to pathological processes, including cerebral edema and severe arachnoiditis that could lead to intracranial hypertension. Besides, 5 out of 9 patients with acute inflammatory CSF pattern had a diagnostic confirmation of an agent associated with chronic meningitis, all of these patients having a subacute course of the disease.

\section{Conclusion}

The presence of immunocompromise related to immunotherapy and neoplasm, as well as changes in alertness, acute inflammatory pattern in CSF, evolution time greater than 28 days, and clinical findings suggestive of intracranial hypertension, confirmed by the presence of hydrocephalus in imaging studies, are associated with in-hospital mortality in patients diagnosed with subacute / chronic meningitis, thus special attention should be paid to cases presenting with these characteristics, with the intention of improving their prognosis.

The absence of a definitive etiological diagnosis, delay at the beginning of treatment, absence of empirical treatment, or other immunocompromising factors such as alcoholism, DM or HIV infection, were not significantly associated with in-hospital mortality.

\section{Bibliography}

1. Helbok Pongpakdee., et al. "Chronic meningitis in Thailand Clinical characteristics, laboratory data and outcome in patients with specific reference to tuberculosis and cryptococcosis". Neuroepidemiology 26.1 (2005): 37-44.

2. Anderson Willoughby. "Chronic meningitis without predisposing illness". Quarterly Journal of Medicine 63.240 (1987): 283-295.

3. $\quad$ Ellner Benett. “Chronic meningitis”. Medicine 55.5 (1976): 341-369.

4. Cho Venna. "Management of acute, recurrent, and chronic meningitides in adults". Neurologic Clinics 28.4 (2010): 1061-1088.

5. Hildebrand Aoun. "Chronic meningitis: Still a diagnostic challenge". Journal of Neurology 250.6 (2003): 653-660.

6. Anderson., et al. "Leptomeningeal and brain biopsy in chronic meningitis". Australian and New Zealand Journal of Medicine 25.6 (1995): 703-706.

7. Helbok., et al. "Chronic Meningitis". Journal of Neurology 256.2 (2009): 168-175.

8. Murthy. "Fungal infections of the central nervous system: The clinical syndromes". Neurology India 55.3 (2007): 221225.

9. Nesseler., et al. "Aseptic meningitis associated with lamotrigine: report of two cases". European Journal of Neurology 14.12 (2007): e3-e4.

10. Colombe., et al. "Chronic meningitis: aetiologies, diagnosis, and treatment". Revue de Médecine Interne 24.1 (2003): 2433.

11. Galldiks., et al. "Komplikationen und diagnostische Grenzen bei chronischer Meningitis". Deutsche Medizinische Wochenschrift 130 (2005): 1633-1636.

12. Schmutzhard E. "Entzündliche Erkrankungen des Nervensystems. Suttgart, New York: Georg Thieme Verlag (2000).

13. Tan TQ. "Chronic meningitis". Seminars in Pediatric Infectious Diseases 14.2 (2003): 131-139.

14. García-Monco. "Central nervous system tuberculosis". Neurologic Clinics 17.4 (1999): 737-759. 
15. CENAVE México (2010).

16. Martínez. "Tuberculosis del sistema nervioso central: conceptos actuals". Revista Mexicana de Neurociencia 1.1 (2000): 3-9.

17. Berenguer J., et al. "Tuberculous meningitis in patients infected with the human immunodeficiency virus". The New England Journal of Medicine 326.10 (1992): 668-672.

18. Dube MP., et al. "Tuberculous meningitis in patients with and without human immunodeficiency virus infection". The American Journal of Medicine 93.5 (1992): 520-524.

19. Klein NC., et al. "Mycobacterial meningitis: Retrospective analysis from 1970 to 1983 ". The American Journal of Medicine 79.1 (1985): 29-34.

20. Ogawa SK., et al. "Tuberculous meningitis in an urban medical center". Medicine (Baltimore) 66.4 (1987): 317-326.

21. Verdon R., et al. "Tuberculous meningitis in adults: a review of 48 cases". Clinical Infectious Diseases 22.6 (1996): 982988.

22. Davis LE., et al. "Tuberculous meningitis in the south-west United States: a community-based study". Neurology 43.9 (1993):1775-1778.

23. Girgis NI., et al. "Tuberculosis meningitis, Abbassia Fever Hospital-Naval Medical Research Unit No.3-Cairo, Egypt, from 1976-1996". The American Journal of Tropical Medicine and Hygiene 58.1 (1998): 28-34.

24. Thwaites GE and Tran TH. "Tuberculous meningitis: many questions, too few answers". Lancet Neurology 4.3 (2005): 160-170.

25. Sutlas PN., et al. "Tuberculous meningitis in adults: a review of 61 cases". Infection 31.6 (2003): 387-391.

26. Thwaites GE., et al. "The influence of HIV infection on clinical presentation, response to treatment, and outcome in adults with tuberculous meningitis". The Journal of Infectious Diseases 192.12 (2005): 2134-2141.

27. Bernaerts A., et al. "Tuberculosis of the central nervous system: overview of neurorradiological findings". European Radiology 13.8 (2003): 1876-1890.

28. Kumar R., et al. "Value of CT scan in the diagnosis of meningitis". Indian Pediatrics 33.6 (1996): 465-468.
29. Morgado C and Ruivo N. "Imaging meningo-encephalic tuberculosis". European Journal of Radiology 55.2 (2005):188192.

30. Nair PP., et al. "MRI pattern of infarcts in basal ganglia regios in patients with tuberculous meningitis". Neurorradiology 51.4 (2009): 221-225.

31. Thwaites GE., et al. "Serial MRI to determine the effects of dexamethasone on the cerebral pathology of tuberculous meningitis". Lancet 360.9342 (2002): 1287-1292.

32. Ceccini D., et al. "Tuberculous meningitis in HIV-infected and non-infected patients: comparison cerebrospinal fluid findings". International Journal of Tuberculosis and Lung Disease 13.2 (2009): 269-271.

33. Katrak SM., et al. "The clinical, radiological, and pathological profile of tuberculous meningitis in patients with and without human immunodeficiency virus infection". Journal of the Neurological Sciences 181.1-2 (2000): 118-126.

34. Marais., et al. "Tuberculous meningitis: a uniformcasedefinitions for use in clinical research". Lancet Infectious Diseases 10.11 (2010): 803-812.

35. Teoh R and Humphries M. "Tuberculous meningitis". In: Lambert HP, editor. Kass handbook of infectious diseases: Infections of the central nervous system. Philadelphia PA: BC Decker (1991): 189-206.

36. Thwaites GE., et al. "Improving the bacteriological diagnosis of tuberculous meningitis". Journal of Clinical Microbiology 42.1 (2004): 378-379.

37. Roos. "Mycobacterium tuberculosis meningitis and other etiologies of the aseptic meningitis syndrome". Seminars in Neurology 20.3 (2000): 329-335.

38. Rock., et al. "CNS tuberculosis: Pathogenesis and clinical aspects". Clinical Microbiology Reviews 21.2 (2008): 243-261.

39. Thwaites GE., et al. "British Infection Society guidelines for the diagnosis and treatment of tuberculosis of the central nervous system in children and adults". Journal of Infection 59.3 (2009):167-187.

40. Rees JR., et al. "The epidemiologocal features of invasive mycotic infections in the San Francisco Bay Area, 1992-1993: results of population-based laboratory active surveillance". Clinical Infectious Diseases 27.5 (1998): 1138-1147. 
41. Rauchway., et al. "Neurologic presentations of fungal infections". Neurologic Clinics 28.1 (2010): 293-309.

42. Bodey G., et al. "Fungal infections in cancer patients: an international autopsy survey". European Journal of Clinical Microbiology and Infectious Diseases 11.2 (1992): 99-109.

43. Sanchez-Portocarrero J., et al. "central nervous system and infection by Candida species". Diagnostic Microbiology and Infectious Disease 37.3 (2000): 169-179.

44. Ginsberg, Kidd. "Chronic and recurrent meningitis". Practical Neurology 8.6 (2008): 348-361.

45. Perfect J., et al. "Clinical practice guidelines for the management of cryptococcal disease: 2010 update by the infectious diseases society of America". Clinical Infectious Diseases 50.3 (2010): 291-322.

46. Anstead Graybill. "Coccidioidomycosis". Infectious Disease Clinics of North America 20.3 (2006): 621-643.

47. Vincent., et al. "The natural history of coccidioidal meningitis". Clinics of Internal Medicine 16.2 (1993): 247-254.

48. Flood., et al. "Neurosyphilis during the AIDS epidemic, SF, 1985-1992". Journal of Infectious Diseases 177.4 (1998): 931-940.

49. Marra., et al. "Cerebrospinal fluid abnormalities in patients with syphilis: association with clinical and laboratory features". Journal of Infectious Diseases 189.3 (2004): 369-376.

50. Ghanem., et al. "Neurosyphilis in a cohort of HIV-infected patients”. AIDS 22.10 (2008): 1145-1151.

51. Castro., et al. "Nontreponemal tests in the diagnosis of neurosyphilis: an evaluation of VDRL y RPR tests". Journal of Clinical Laboratory Analysis 22.4 (2008): 257-261.

52. Davis Schmitt. "Clinical significance of cerebrospinal fluid tests for neurosyphilis”. Annals of Neurology 25.1 (1989): 50-55.

53. Smikle James and Prabhakar. "Diagnosis of neurosyphilis: a critical assessment of current methods". Southern Medical Journal 81.4 (1988): 452-454.
54. Wormser., et al. "The clinical assessment, treatment, and prevention of Lyme disease, human granulocytic anaplasmosis, and babesiosis: Clinical practice guidelines by the Infectious Disease Society of America". Clinical Infectious Diseases 43.9 (2006): 1089-1134.

Volume 2 Issue 10 October 2019

(C) All rights are reserved by Rodríguez-Leyva I., et al. 